\title{
Isotopic effect in experiments on lower hybrid current drive in the FT-2 tokamak
}

\author{
S.I. Lashkul, A.Yu. Stepanov, A.B. Altukhov, A.D. Gurchenko, E.Z. Gusakov, V.V. Dyachenko, \\ L.A. Esipov, M.A. Irzak, M.Yu. Kantor, D.V. Kouprienko, A.N. Saveliev and S.V. Shatalin* \\ Ioffe Institute, Politekhnicheskaya 26, St.Petersburg, Russia \\ *St.Petersburg State Polytekhnical University, Politekhnicheskaya 29, St.Petersburg, Russia
}

\section{Result of the LHCD efficiency study}

The most efficient method for sustaining the quasisteady plasma current by means of lower hybrid (LH) waves can be implemented only at relatively low plasma densities not exceeding a certain density limit, $n_{D L}$. The existence of the density limit for LH current drive (LHCD) was attributed to various mechanisms: linear absorption by ions, which increases as the density tends to the resonance LH value; collision losses; scattering by drift turbulence; parametric instabilities; etc. The density limit effect has been studied and discussed for several past decades; however, it has not received comprehensive physical explanation [1].

In the present work the main attention is paid to investigation of current drive by lower hybrid waves on the FT-2 $\left(R=0.55 \mathrm{~m}, a=0.08 \mathrm{~m}, B_{T} \leq 3 \mathrm{~T}, I_{p}=19 \div 40 \mathrm{kA}\right.$, $f_{0}=920 \mathrm{MHz}$ ) tokamak. The results of experiments carried out at the FT-2 tokamak allow us to conclude that the most probable reason for LHCD termination in both hydrogen and deuterium plasmas at a relatively low plasma current of $I=22 \mathrm{kA}$ and, accordingly, a low electron temperature is an additional reduction in $T_{e}$ at the periphery of the plasma column during the LH pulse [2]. This results in a lower threshold for the parametric decay $\left(\sim T_{e} / n_{e}\right)$ of the pumping wave. The parametric decay satellites, the frequencies of which are down-shifted, $\left(f_{\text {sat }}=\right.$ $\left.f_{0}-k f_{C i}\right),(k=1,2,3 \ldots)$, are slowed down more than the pumping wave. Therefore, the plasma density, at which linear conversion of slowed down satellite waves occurs, is lower than that for the pumping wave; hence, the LH wave after parametric decay can be absorbed by ions even at the plasma periphery, without penetrating into the plasma column. At a higher plasma current $\left(I_{O H}=32 \mathrm{kA}\right)$ and higher electron temperature $\left(T_{e 0}=600 \mathrm{eV}\right)$, the density at which the LHCD in hydrogen plasma terminates is close to the resonance value $\left(n_{D L}{ }^{H} \approx n_{L H}{ }^{H} \approx 3.510^{19} \mathrm{~m}^{-3}\right)$. After the plasma density reaches this value, the interaction of the LH wave with electrons is replaced with direct absorption by ions, when $\omega_{0} \sim \omega_{\mathrm{pl}, \mathrm{i}}$. As a result of the experimental study of the influence of the plasma isotopic composition on the LHCD efficiency, it is established that the efficiency $\eta_{C D}{ }^{D}$ in deuterium plasma on FT-2 is higher than in hydrogen plasma $\eta_{C D}{ }^{H}$ [2]. As it is seen from Fig. $1\left(I_{O H}=32 \mathrm{kA}\right)$, within the density range from $<n_{e}>==10^{19} \mathrm{~m}^{-3}$ to $<n_{e}>=2.510^{19} \mathrm{~m}^{-3}$ in deuterium, we have $\eta_{C D}{ }^{D} \approx 0.4 \mathrm{~A} \mathrm{~m}^{-2} \mathrm{~W}^{-1}$, whereas in hydrogen plasma, this value is appreciably smaller, $\eta_{C D} \approx 0.3 \mathrm{~A} \mathrm{~m}^{-2}$ $\mathrm{W}^{-1}$. The resonance value $n_{L H}$ of the density for deuterium at $N \mathrm{II} \approx 2$ is substantially higher, $n_{L H}{ }^{D} \approx 10^{20} \mathrm{~m}^{-3} \geq$ $n_{D L}{ }^{D} \approx 4.510^{19} \mathrm{~m}^{-3}$. So, the obtained value $n_{D L}{ }^{D} \approx 4.5$ $10^{19} \mathrm{~m}^{-3}$ is smaller by more than one-half of the $n_{L H}{ }^{D}$.
Nevertheless, in contrast to hydrogen plasma, the density at which the sharp increase in the high-energy chargeexchange (CX) atomic flux (Fast Neutral (FN) with $\left.E_{C X}=1575 \mathrm{eV}\right)<n_{e}>_{F N} D \approx 510^{19} \mathrm{~m}^{-3}$, takes place proved to be higher than $n_{D L}{ }^{D}$. There is an appreciable gap between the values of $n_{D L}{ }^{D}$ and $\left\langle n_{e}\right\rangle_{F N}{ }^{D}$. Apparently, the main reason for LHCD termination in this case is the parametric decay of the pumping wave. The experimental results confirm that parametric processes intensify with increasing density during the RF pulse (see Fig. 3 in [2]). Nevertheless, we cannot exclude the influence of a substantial slowing down (up to $\mathrm{NI} \approx 10$ ) of the pumping wave as it propagates into the plasma column. The experimental evidence of influence of isotopic effect on characteristics of the ion heating observed at $\left\langle n_{e}\right\rangle$ rise and after LHCD termination, which demonstrates Fig. 2 for H/D plasmas.

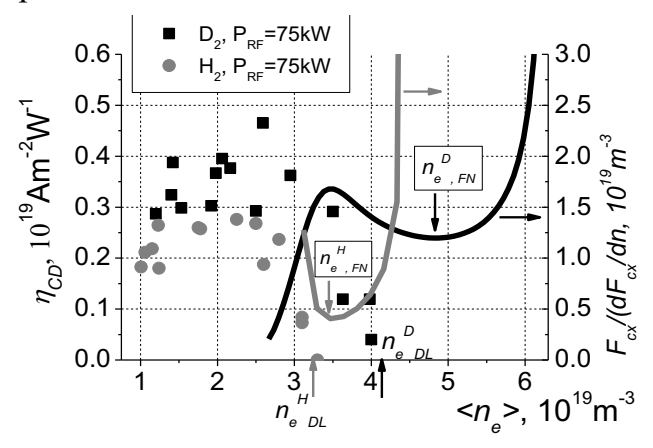

Fig. 1. Experimental values of the LHCD efficiency $\eta_{C D}=I_{R F}^{N}\left\langle n_{e}\right\rangle$ and the quantity $F_{C X} /\left(d F_{C X} / d n_{e}\right)$ vs. average densities of hydrogen plasma (gray circles and curve) and deuterium plasma (black squares and curve). The minimum values of $n_{e}{ }^{D}, F N$ and $n_{e}{ }^{H}, F N$ are marked with arrows

A significant part of the LHCD experimental studies on FT-2 were performed at relatively low densities of $<n_{e}>=10^{19} \mathrm{~m}^{-3}$, when, the parametric decay of the pumping wave is absent and the highest LHCD efficiency is observed. It is established that, at relatively large values of the plasma current $\left(I_{O H}=32 \div 35 \mathrm{kA}, P_{R F} \approx 100 \mathrm{~kW}\right.$, $T_{e 0} \approx 550 \div 600 \mathrm{eV}$ ), the quantities $\eta_{C D}$ and $I_{R F}{ }^{N}$ and their dependences on the plasma parameters are close to those obtained at large tokamaks [2].

\section{Parametric processes intensify \\ with increasing plasma density}

Parametric process on the periphery of the plasma column are considered as the main mechanism of growth $N \mathrm{NI}$ resulting in a significant reduction of LHCD efficiency and depletion of the pump wave power due to the absorption of ions in the peripheral plasma [3,4]. The 


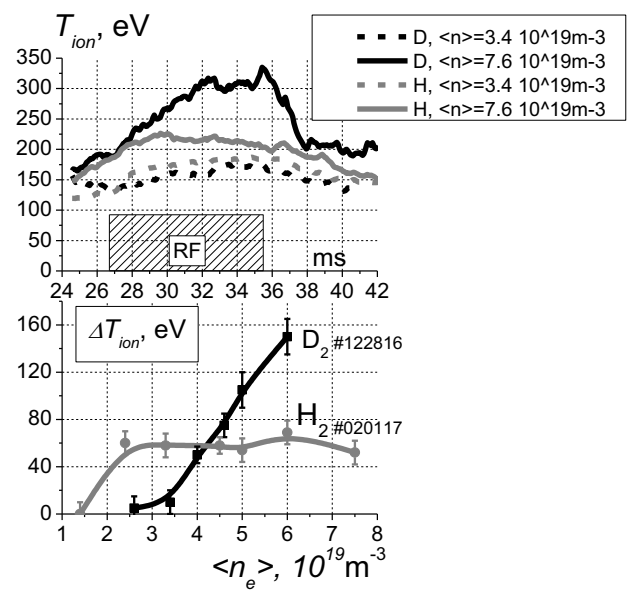

Fig. 2. (a) Experimental values of the $T_{i o n}(t)$ at $P_{R F}=90 \mathrm{~kW}$ for $\mathrm{H} / \mathrm{D}$ plasmas with initial density $<n_{e}>$ increase. (b) Dependence of the increment of the ion temperature vs $\left\langle n_{e}>\right.$ of H/D plasmas, $\Delta T_{i o n}\left(<n_{e}>\right) . \Delta E_{C X}=(364-1058) \mathrm{eV}$ is energy neutral particle spectral interval used for $T_{\text {ion }}$ definition

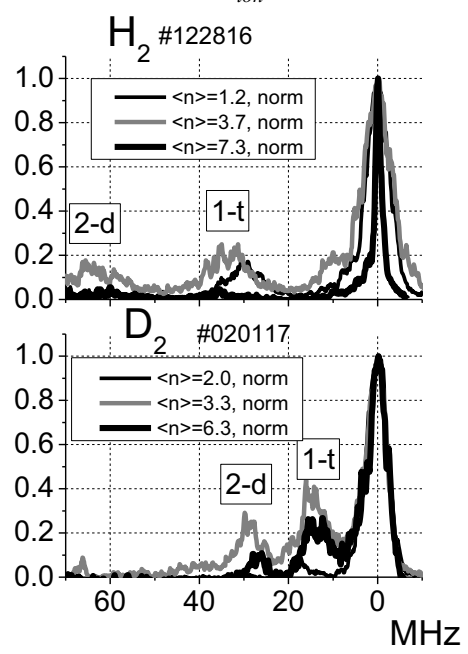

Fig. 3. The normalized intensity of peaks of the LHW pump wave spectra; 1-t and 2-d IC satellites measured by RF probe in $\mathrm{H} / \mathrm{D}$ plasmas at different densities, $<\mathrm{n}>, 10{ }^{19} \mathrm{~m}^{-3}$

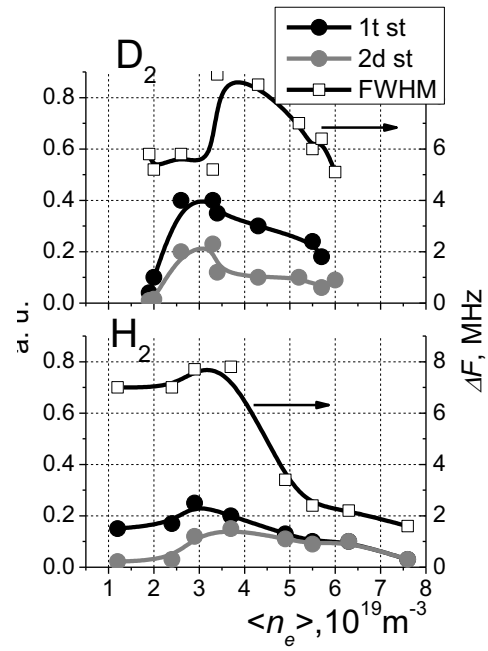

Fig. 4. The normalized intensity of the first, 1-t, and the second, 2-d, satellites of the LHW pump wave. $\Delta F$ - frequency width (FWHM) $\Delta F, \mathrm{MHz}$ of the broadened pump wave spectral broadening of the pump wave at development of PI is due to the excitation by the pump wave ion-sound (IS) and ion-cyclotron (IC) quasimodes.

To assess the impact of isotopic effect on the development of parametric processes on the tokamak FT-2 a comparative measurements were carried out using the movable RF probe-antenna placed in the poloidal limiter shadow at the poloidal angle $\theta=310^{\circ}$, which corresponds to the ICR in the plane of a large radius $\mathrm{R}=0.6 \mathrm{~m}$. Normalized spectra of the LH pump wave measured by the RF probe at different densities of the H/D plasma are shown in Fig. 3. It is noted that the increase in density results in a significant change in the intensity of IC satellites as well as the frequency width (FWHM) $\Delta F, \mathrm{MHz}$ of the broadened pump wave. As shown in Fig. 4, maximum values for these dependencies as a function of $\left\langle n_{e}\right\rangle$ are achieved with plasma densities of $\left\langle n_{e}\right\rangle \sim 3 \div 410^{19} \mathrm{~m}^{-3}$. Under these conditions, according to Fig. 1 , the efficiency generation of LHCD is reduced almost to 0 .

Characteristically, those IC satellites in deuterium plasma appear at a density higher, than the density in hydrogen plasma. With increasing density its level is approximately 2 times higher than the level of the same dependencies measured in hydrogen plasma. This fact, in particular, may indicate a significantly larger pump wave depletion effect, when large fraction of LHW power falls in sidebands. Shift down frequencies $\left(f_{\text {sat }}=f_{0}-k f_{C i}\right)$, $(k=1,2,3 \ldots)$, at substantial rise of $N I I$ for $f_{\text {sat }}$ could result in additional heating of the ions on periphery of discharge. Most of the LHW power of $f_{0}$ in the course of propagation along the magnetic field lines with the gradual penetration in the plasma column reaches it central regions. During such penetration of LHW the effect of spectral broadening $\Delta F$ (Fig. 4) and additional slowdown result in decrease of the efficiency generation of the LHCD. With increasing density, more effective additional heating of the deuteron are observed Fig. 1, 2 .

These and other experimental data presented in the report allows the use of isotope effect to conduct a comparative analysis for obtaining more information about the mechanisms affecting the efficiency of generation of LHCD and ion heating.

This work is executed at partial financial support by Russian Science Foundation grant 17-12-01110

\section{References}

1.M. Goniche, L. Amicucci, Y. Baranov et al. Lower hybrid current drive for the steady-state scenario. // Plasma Phys. Controlled Fusion 52, 124031 (2010).

2. S.I. Lashkul, A.B. Altukhov, A.D. Gurchenko et al. Isotopic Effect in Experiments on Lower Hybrid Current Drive in the FT-2 Tokamak. // Plasma Physics Reports, Plasma Physics Reports, 2015, Vol. 41, No. 12, pp. 990-996.

3. R. Cesario, L. Amicucci, A. Cardinali et al. Spectral broadening of parametric instability in lower hybrid current drive at a high density. // Nucl. Fusion 54 (2014) 043002 (18pp).

4. M. Goniche1, V. Basiuk1, J. Decker et al. Lower hybrid current drive at high density on Tore Supra. // Nucl. Fusion 53 (2013) 033010 (9pp). 